\title{
CIRCUMSCRIBING CUBES OF A HYPERELLIPSOID ${ }^{1}$
}

\section{DOUGLAS H. TAYLOR}

1. Introduction. Let $C$ be a compact convex subset, with nonempty interior, of Euclidean $n$-space, $R^{n}$. To each unit vector $v$, let $w(v)$ be the distance between the supporting hyperplanes of $C$ normal to $v$. If $\mathrm{SO}(n)$ is the group of rotations in $R^{n}$, then $w$ induces a function $W: \mathrm{SO}(n) \rightarrow R^{n}$ as follows,

$$
W\left(\boldsymbol{v}_{1}, \boldsymbol{v}_{2}, \cdots, \boldsymbol{v}_{n}\right)=\left(w\left(\boldsymbol{v}_{1}\right), w\left(\boldsymbol{v}_{2}\right), \cdots, w\left(\boldsymbol{v}_{n}\right)\right) .
$$

The inverse image of the diagonal in $R^{n}$ will consist of all orthogonal $n$-frames made up of vectors normal to the faces of a circumscribing cube about $C$. Each element of this set will be called a rotation that determines a circumscribing cube around $C$. In 1942, S. Kakutani [6] conjectured that this set was nonempty for any convex body $C$. This was proved by H. Yamabe and Z. Yujobo in 1950, [10]. Proof of Kakutani's conjecture when $n=3$ may be found in [5] and [6]; when $n=4$ in [5] and [7]. Therefore there exists a circumscribing cube around every convex body.

These results were strengthened by S. S. Cairns in 1959, [1] and [2]. The set of all circumscribing boxes around $C$ was made into a topological space, with subspace $K$ consisting of the circumscribing cubes. The main result in [2] is that the dimension of $K$ is greater than or equal to the dimension of $\mathrm{SO}(n-1)$. Also contained there is the conjecture that $K$ always contains a subspace homeomorphic to $\mathrm{SO}(n-1)$. This was proved when $n=3,[2, \mathrm{p} .100]$. This paper is an attempt to investigate this conjecture for $n>3$. The approach taken is to investigate the circumscribing cubes of the hyperellipsoid,

$$
E=\left\{\left(x_{1}, x_{2}, \cdots, x_{n}\right) \mid \sum_{1}^{n}\left(\frac{x_{i}}{a_{i}}\right)^{2} \leqq 1\right\} .
$$

In $\$ 2$ we show that results about circumscribing cubes of centrally symmetric convex bodies are valid for arbitrary convex bodies, (Theorem 2.1). If $C$ is centrally symmetric then every rotation that determines a circumscribing cube about $C$ also determines a set of equal orthogonal radii of another convex body, $C^{*}$, (Theorem 2.2). So questions about circumscribing cubes are equivalent to questions about equilateral, orthogonal, inscribed frames.

Received by the editors December 2, 1965.

1 This research was done under NSF grant GP 730 . 
In $\S \S 3-5$ only the hyperellipsoid is discussed. Theorem 3.1 states that every circumscribing cube of $E$ has the same edge-length. This edge-length is equal to $2\left(1 / n \sum_{1}^{n} a_{i}^{2}\right)^{1 / 2}$. Theorem 3.2 characterizes the set of unit vectors normal to faces of circumscribing cubes around $E$. This set of unit vectors, as a subspace of the unit $(n-1)$-sphere, is a product of spheres, $S^{p} \times S^{q}$, where $p+q=n-2$, (Theorem 4.1). Results concerning the space of rotations determining circumscribing cubes of $E$ are found in $\$ 5$, (Theorem 5.1).

The author wishes to express his gratitude to Professor Stewart S. Cairns for his valuable suggestions and assistance.

2. General results. The theorems of this section pertain to studying the circumscribing cubes of an arbitrary convex body, C. According to Theorem 2.1 we may assume that $C$ is centrally symmetric. By Theorem 2.2 we can equivalently study equilateral and orthogonal radii.

Let $R^{n}$ be Euclidean $n$-space with $\mathrm{x} \cdot \mathrm{y}$ denoting the inner product. The dual of $\mathrm{x} \in R^{n}$ is the closed half space $x^{*}=\{y \mid x \cdot y \leqq 1\}$. If $C$ is any convex body containing the origin then the dual of $C$ is the convex body $C^{*}=\bigcap_{x^{*}}(x \in C)$. The distance function of $C, d: R^{n} \rightarrow R$ is defined by

$$
d(x)=\inf \{r>0 \mid x \in r(C)\},
$$

where $r(C)$ is the convex body obtained by multiplying each vector in $C$ by $r$. The support function of $C, s: R^{n} \rightarrow R$, is the distance function of $C^{*}$. If $C$ is a closed convex body containing the origin then $C^{* *}=C\left[4\right.$, p. 26]. Therefore the distance (support) function of $C^{*}$ is the support (distance) function of $C$. The width of $C$ in the direction $\mathbf{x}(|\mathbf{x}|=1)$ is $s(\mathbf{x})+s(-\mathbf{x})$.

TheOREM 2.1. To each convex set, $C$, there corresponds a centrally symmetric convex set, $C^{\prime}$, such that $C$ and $C^{\prime}$ have the same width in any direction.

Let $C^{\prime}=1 / 2(C-C)$, i.e. $C^{\prime}$ is the set of all vectors of the form $1 / 2(y-z)$ where $y$ and $z$ range over all points of $C$. $C^{\prime}$ is a centrally symmetric convex body with support function

$$
S^{\prime}(\mathbf{x})=1 / 2(S(\mathbf{x})+S(-\mathbf{x})) \quad[4, \text { p. 101] }
$$

Since $S^{\prime}(\mathrm{x})+S^{\prime}(-\mathrm{x})$ is equal to $S(\mathrm{x})+S(-\mathrm{x})$, the theorem follows.

THEOREM 2.2. There is a one-to-one correspondence between the circumscribing cubes of $C$ and sets $d_{1}, d_{2}, \cdots, d_{n}$ of equal and mutually orthogonal diameters of $\left(C^{\prime}\right)^{*}$. 
This is immediate from 2.1 and the fact that the support function of a convex set is the distance function of its dual.

3. Circumscribing cubes of the hyperellipsoid. Let $E$ be the set of all points $\left(x_{1}, x_{2}, \cdots, x_{n}\right) \in R^{n}$ such that $\sum_{1}^{n}\left(x_{i} / a_{i}\right)^{2} \leqq 1$. If the $a_{i}$ are all equal then $E$ is a solid ball and each of its circumscribing cubes has the same edge-length. The following theorem shows that this is a property shared by all hyperellipsoids.

THEOREM 3.1. Every circumscribing cube about $E$ has edge length equal to $2\left(1 / n \sum_{1}^{n} a_{i}^{2}\right)^{1 / 2}$.

We first compute the support function, $s$, of $E$. By definition, if $|\boldsymbol{v}|=1, s(\boldsymbol{v})=\boldsymbol{v} \cdot \boldsymbol{p}$ where the tangent plane of $E$ at $\boldsymbol{p}$ is normal to $\boldsymbol{v}=\left(v_{1}, v_{2}, \cdots, v_{n}\right)$. Since the $i$ th coordinate of $\boldsymbol{p}$ is $a_{i}^{2} v_{i} / k$ where $k=\left(\sum_{1}^{n}\left(a_{i} e_{i}\right)^{2}\right)^{1 / 2}$ it follows that

$$
s(v)=v \cdot p=\frac{1}{k} \sum_{1}^{n}\left(a_{i} v_{i}\right)^{2}=\left(\sum_{1}^{n}\left(a_{i} v_{i}\right)^{2}\right)^{1 / 2} .
$$

So the dual of $E$ is the hyperellipsoid $\sum\left(a_{i} x_{i}\right)^{2} \leqq 1$.

Let $\left(x_{i j}\right), i, j=1,2, \cdots, n$ be an orthogonal matrix, whose $k$ th column is $x_{k}$. The following equation implies that the sum of the squares of the edge-lengths of any circumscribing box about $E$ is a constant.

$$
\sum_{j} s^{2}\left(x_{j}\right)=\sum_{j} \sum_{i} a_{i}^{2} x_{i j}^{2}=\sum_{i} a_{i}^{2} \sum_{j} x_{i j}^{2}=\sum_{i} a_{i}^{2} .
$$

If $\mathbf{x}_{j}, j=1,2, \cdots, n$ are normal to the sides of a circumscribing cube with edge-length $2 e$ then

$$
\sum_{j} s^{2}\left(x_{j}\right)=n \cdot e^{2}=\sum_{i} a_{i}^{2} .
$$

Therefore $e=\left((1 / n) \sum_{j} a_{i}^{2}\right)^{1 / 2}$. From now on we reserve the symbol $e$ for this quantity.

Theorem 3.1 states that if $v$ is a unit vector normal to the face of a circumscribing cube of $E$ then $s(v)=e$. The following theorem is the converse; if $s(v)=e$ then $v$ is normal to the face of a circumscribing cube about $E$.

THEOREM 3.2. Let $\mathrm{x}_{1}$ be a unit vector satisfying $s\left(\mathrm{x}_{1}\right)=e$; then there is an orthonormal frame $\left(\mathbf{x}_{1}, \mathbf{x}_{2}, \cdots, \mathbf{x}_{n}\right)$ with $s\left(\mathbf{x}_{i}\right)=e, i=1,2, \cdots, n$.

Let $E^{\prime}$ be the projection of $E$ into the hyperplane normal to $x_{1}$. 
Let $b_{1}, b_{2}, \cdots, b_{n-1}$ be the radii of the axes of $E^{\prime}$. From (1)

$$
\sum_{1}^{n} s^{2}\left(x_{i}\right)=\sum_{1}^{n-1} b_{i}^{2}+e^{2}=\sum_{1}^{n} a_{i}^{2}=n e^{2}
$$

and

$$
\frac{1}{n-1} \sum_{1}^{n-1} b_{i}^{2}=\frac{1}{n} \sum_{1}^{n} a_{i}^{2} .
$$

Therefore circumscribing cubes of $E^{\prime}$ have the same edge length as those of $E$. Also, there must be a vector $x_{2}$ normal to $x_{1}$ such that $s\left(x_{2}\right)=e$. Projecting $E^{\prime}$ onto the space normal to $\mathbf{x}_{2}$ and repeating the argument above proves the theorem. This theorem establishes the existence of a circumscribing cube about $E$.

Because of the duality between distance and support functions, mentioned in $\$ 2$, we have the following corollary.

Corollary 3.3. If $\boldsymbol{r}$ is a radius of $E$; then $\boldsymbol{r}$ is an element of an inscribed equilateral frame if and only if $|r|=\left((1 / n) \sum\left(1 / a_{i}\right)^{2}\right)^{-1 / 2}$.

Let $d$ be the distance function of $E$ and hence the support function of $E^{*}$. Let $\left(\boldsymbol{r}_{1}, \boldsymbol{r}_{2}, \cdots, \boldsymbol{r}_{n}\right)$ be an inscribed equilateral frame and $\left(v_{1}, v_{2}, \cdots, v_{n}\right)$ the corresponding frame of unit vectors. So $\left(v_{1}, v_{2}, \cdots, v_{n}\right)$ determines a circumscribing cube about $E^{*}$ and by $3.1 d\left(v_{i}\right)=\left((1 / n) \sum\left(1 / a_{j}\right)^{2}\right)^{1 / 2}$. But if $v$ is a unit vector $d(v)$ is the reciprocal of the radius determined by $v$. Therefore $\left|r_{i}\right|=((1 / n)$ $\left.\sum\left(1 / a_{i}\right)^{2}\right)^{-1 / 2}, i=1,2, \cdots, n$.

4. Intersection of a sphere and hyperellipsoid. In $\$ 3$ the unit vectors normal to sides of circumscribing cubes were characterized as those vectors $\boldsymbol{v}$ satisfying $s(\boldsymbol{v})=e$. In this section, the topology of this set of vectors as a subspace of the unit $(n-1)$-sphere is determined. For this consider $F: S^{n-1} \rightarrow R$ defined by

$$
F(v)=s^{2}(v)=\sum_{1}^{n}\left(a_{i} v_{i}\right)^{2} .
$$

The set we wish to investigate is $F^{-1}\left(e^{2}\right)$ which is the intersection of $S^{n-1}$ and the hyperellipsoid $\sum_{1}^{n}\left(a_{i} x_{i}\right)^{2}=e^{2}$. This intersection can be determined by applying Morse's critical point theory to the function $F$.

So that the critical points will be nondegenerate we assume that the $a_{i}$ 's are all distinct and that $a_{i}<a_{i+1}, i=1,2, \cdots, n-1$. Let $L_{r}$ be $F^{-1}(-\infty, r)$. The main theorem of $[8$, p. 2] states the following. Sup- 
pose that $c$ is the only critical value in $[a, b]$ and that $p_{1}, p_{2}, \cdots, p_{m}$ are the critical points at this level with indices $k_{1}, k_{2}, \cdots, k_{m}$, respectively; then $L_{b}$ is diffeomorphic to $L_{a}$ with $m$ handles of index $k_{i}$ smoothly and disjointly attached. The function $F$ of equation (2) has critical points $(0, \cdots, \pm 1, \cdots, 0)$ with indices $(i-1)$ where the \pm 1 occurs at the $i$ th coordinate. Therefore in dimension 3

$$
\begin{array}{ll}
L_{r}=\varnothing & \text { when } r<a_{1}^{2}, \\
L_{r}=B_{1} \cup B_{2} & \text { when } a_{1}^{2}<r<a_{2}^{2}, \\
L_{r}=A^{2} & \text { when } a_{2}^{2}<r<a_{3}^{2} \text { and } \\
L_{r}=S^{2} & \text { when } a_{3}^{2}<r .
\end{array}
$$

Here $B_{1}$ and $B_{2}$ are two-dimensional disks and $A^{2}$ is a two-dimensional annulus. When $n=4$

$$
\begin{array}{ll}
L_{r}=\varnothing & \text { when } r<a_{1}^{2}, \\
L_{r}=C_{1} \cup C_{2} & \text { when } a_{1}^{2}<r<a_{2}^{2}, \\
L_{r}=A^{3} & \text { when } a_{2}^{2}<r<a_{3}^{2}, \\
L_{r}=C_{1}^{\prime} \cup C_{2}^{\prime} & \text { when } a_{3}^{2}<r<a_{4}^{2}, \\
L_{r}=S^{3} & \text { when } a_{4}^{2}<r,
\end{array}
$$

where $C_{1}, C_{2}, C_{1}^{\prime}$, and $C_{2}^{\prime}$ are three dimensional disks and $A^{3}$ a three dimensional annulus. In each case the set $F^{-1}\left(e^{2}\right)$ is the boundary of the manifold $L_{r}$. Therefore when $n=3, F^{-1}\left(e^{2}\right)$ is a pair of 1 -spheres and when $n=4, F^{1}\left(e^{2}\right)$ is either a pair of 2 -spheres or a torus. In each case $F^{-1}\left(e^{2}\right)$ is a product of spheres and is dependent upon the position of $e^{2}$ among the $a_{1}^{2}, a_{2}^{2}, \cdots, a_{n}^{2}$. That this is always so, is the result of the following theorem.

TheOREM 4.1. If $a_{k}<e<a_{k+1}$ then $F^{-1}\left(e^{2}\right)$ is homeomorphic to $S^{k-1}$ $\times S^{n-k-1}$.

Let $S^{k-1}$ be the unit sphere in the $k$-dimensional subspace spanned by the $a_{1}, a_{2}, \cdots, a_{k}$ axes of $E$. The distance between the supporting hyperplanes normal to any vector of $S^{k-1}$ is at most $2 a_{k}$ and therefore is too small to determine faces of a circumscribing cube. If $S^{n-k-1}$ is the unit sphere in the subspace spanned by the $a_{k+1}, a_{k+2}, \cdots, a_{n}$ axes, then these vectors determine supporting hyperplanes too far apart to be faces of a circumscribing cube.

For every $\boldsymbol{p} \in S^{k-1}$ and $\boldsymbol{q} \in S^{n-k-1}$ let $H(\boldsymbol{p}, \boldsymbol{q})$ be the two dimensional subspace spanned by the origin $\boldsymbol{p}$ and $\boldsymbol{q}$. The points $\boldsymbol{p}$ and $\boldsymbol{q}$ 
determine a geodesic quarter-circle $(\boldsymbol{p}, \boldsymbol{q})$ on $S^{n-1}$. If $E^{1}$ is the projection of $E$ into $H(\boldsymbol{p}, \boldsymbol{q})$ then for $\boldsymbol{v} \in(\boldsymbol{p}, \boldsymbol{q}), s(\boldsymbol{v})$ will be the distance between the supporting lines of $E^{1}$ normal to $\boldsymbol{v}$. If $\boldsymbol{p}$ and $\boldsymbol{q}$ lie on the minor and major axis of $E^{1}$, then $s$ will be strictly increasing on $(\boldsymbol{p}, \boldsymbol{q})$ and therefore must assume the value $e$ at exactly one point, $P(\boldsymbol{p}, \boldsymbol{q})$. If the major axis of $E^{1}$ is contained in the arc $(\boldsymbol{p}, \boldsymbol{q})$, let $\boldsymbol{q}^{\prime}$ be the reflection of $q$ about the major axis. Since $S(q)$ is too large to determine faces of a cube the same must be true of every vector in the $\operatorname{arc}\left(\boldsymbol{q}^{\prime}, \boldsymbol{q}\right)$. However $S$ will be strictly increasing on the $\operatorname{arc}\left(\boldsymbol{p}, \boldsymbol{q}^{\prime}\right)$ and again will attain the value $e$ at just one point, $P(\boldsymbol{p}, \boldsymbol{q})$.

Moreover the points $P(\boldsymbol{p}, \boldsymbol{q})$ are distinct for distinct pairs $\left(\boldsymbol{p}_{\mathbf{1}}, \boldsymbol{q}_{\mathbf{1}}\right)$ and $\left(\boldsymbol{p}_{2}, \boldsymbol{q}_{2}\right)$. Suppose $\boldsymbol{p}_{1} \neq \boldsymbol{p}_{2}$, while $\boldsymbol{q}_{1}=\boldsymbol{q}_{2}$; then $H\left(\boldsymbol{p}_{1}, \boldsymbol{q}\right)$ and $H\left(\boldsymbol{p}_{2}, \boldsymbol{q}\right)$ will intersect on the line from the origin through $\boldsymbol{q}$ and $P\left(\boldsymbol{p}_{1}, \boldsymbol{q}\right)$ $\neq P\left(\boldsymbol{p}_{2}, \boldsymbol{q}\right)$. The same argument applies when $\boldsymbol{p}_{1}=\boldsymbol{p}_{2}$ and $\boldsymbol{q}_{1} \neq \boldsymbol{q}_{2}$. Suppose now that $\boldsymbol{p}_{1} \neq \boldsymbol{p}_{2}$ and $\boldsymbol{q}_{1} \neq \boldsymbol{q}_{2} . H\left(\boldsymbol{p}_{1}, \boldsymbol{q}_{1}\right)$ and $H\left(\boldsymbol{p}_{2}, \boldsymbol{q}_{2}\right)$ intersect only at the origin, because suppose $t \neq 0$ belonged to both of them. Then the line determined by the origin and $t$ when projected in to the subspace spanned by $a_{1}, a_{2}, \cdots, a_{k}$ would have to have both $\boldsymbol{p}_{1}$ and $\boldsymbol{p}_{2}$ in its image. Therefore the set of all unit vectors satisfying $s(\boldsymbol{v})=e$ is homeomorphic to $S^{k-1} \times S^{n-k-1}$. This $S^{k-1} \times S^{n-k-1}$ separates $S^{n-1}$ into a $D^{k} \times S^{n-k-1}$ on which $s$ is too small and an $S^{k-1} \times D^{n-k}$ on which $s$ is too large, $\left[3\right.$, p. 25]. $D^{k}$ and $D^{n-k}$ are $k$ and $n-k$ disks.

Because of the duality between distance and support functions and because the dual of a hyperellipsoid is a hyperellipsoid the following corollary is immediate.

COROLlary 4.2. The locus of the endpoints of the set of equal and orthogonal diameters of $E$ is homeomorphic to $S^{k-1} \times S^{n-k-1}$.

THEOREM 4.3. The locus of the contact points of $E$ with the faces of its circumscribing cubes is the intersection of $E$ with the hyperellipsoid,

$$
\sum\left(x_{i} / b_{i}\right)^{2}=1 / e^{2} \quad \text { where } b_{i}=a_{i}^{2}, \quad i=1, \cdots, n .
$$

$\left(z_{1}, z_{2}, \cdots, z_{n}\right)$ will be a contact point if and only if the tangent plane, $\sum_{1}^{n} z_{i} x_{i} / a_{i}^{2}=1$, has distance from the origin equal to $e$. That is, if and only if

$$
\left(\sum z_{i}^{2} / a_{i}^{4}\right)^{-1 / 2}=e \quad \text { or } \quad \sum z_{i}^{2} / a_{i}^{4}=1 / e^{2} .
$$

Conjecture. This locus is also a product of spheres.

5. Rotations that determine circumscribing cubes. The space of circumscribing cubes of a convex body, $C$, was defined in $[2$, p. 92]. 
An equivalent definition is the following. Let $V_{n k}$ be the Stiefel manifold of orthogonal $k$-frames in Euclidean $n$-space, [9, p. 33]. With $\pi_{k}: V_{n k} \rightarrow V_{n k-1}$ defined by

$$
\pi_{k}\left(v_{1}, v_{2}, \cdots, v_{k}\right)=\left(v_{1}, v_{2}, \cdots, v_{k-1}\right)
$$

we obtain the sequence

$$
V_{n n} \rightarrow V_{n n-1} \rightarrow \cdots \rightarrow V_{n 2} \rightarrow V_{n 1} .
$$

$V_{n n}$ is the orthogonal group; $V_{n n-1}$, the group of rotations and $V_{n 1}$ the $(n-1)$-sphere. Let $W_{n k}$ consist of all those $k$-frames of vectors normal to the faces of circumscribing cubes. We refer to $W_{n n-1}$ as the space of rotations that determine circumscribing cubes. The space of circumscribing cubes is the quotient space obtained by identifying all elements of $W_{n n-1}$ that determine the same cube, [2, p. 93].

THEOREM 5.1. If $n>3$ and if $E$ is a hyperellipsoid satisfying

$$
a_{1}<e<a_{2}<a_{3}<\cdots<a_{n},
$$

then $W_{n n-1}$ is homeomorphic to $2^{n-1}$ disjoint copies of $V_{n-1, n-2}$.

Consider the sequence

$$
W_{n n} \rightarrow W_{n n-1} \rightarrow \cdots \rightarrow W_{n 2} \rightarrow W_{n 1},
$$

where the mappings are the restrictions of $\pi_{k}$ to $W_{n k}$. By Theorem 4.1, $W_{n 1}$ is homeomorphic to $S^{0} \times S^{n-2}$, the disjoint union of two $(n-2)$-spheres. According to Theorem 3.2, the orthogonal complement of each element in $W_{n 1}$ must intersect $W_{n 1}$. Again by Theorem 4.1 , this intersection must be a product of spheres, $S^{p} \times S^{q}$ where $p+q=n-3$. Since $W_{n 1}$ is not connected, this intersection is not connected and $p=0$ and $q=n-3>0$. Therefore $W_{n 2}$ is homeomorphic to four disjoint copies of $V_{n-1,2}$. Now consider the orthogonal complement of each element in $W_{n 2}$ and apply Theorem 4.1 again. The fiber over each point this time consists of a pair of $(n-4)$-spheres. Repeating this process back to $W_{n n-1}$ proves the theorem.

CoRollaRY 5.1. If $n=5$ or 9 then $W_{n n-1}$ is homeomorphic to $2^{n-1}$ copies of $S^{n-1} \times \mathrm{SO}(n-1)$.

By [9, Corollary 8.18 , p. 42], if $n=4$ or $8, V_{n n-1} \rightarrow S^{n-1}$ is a product bundle. Since the fiber is $\mathrm{SO}(n-1), V_{n n-1}$ is homeomorphic to $S^{n-1} \times \mathrm{SO}(n-1)$.

If $n=3, W_{32}$ is homeomorphic to eight copies of $V_{21}=S^{1}$. This is due to the fact that the fiber of $W_{32} \rightarrow W_{31}$ is $S^{0} \times S^{0} . W_{31}$ is the disjoint union of two 1-spheres and each of these 1-spheres is lifted back 
to $W_{32}$ in four ways. If $\left(v_{1}, v_{2}, v_{3}\right)$ is a frame of vectors all belonging to a single component, $S^{1}$, of $W_{32}$, then $\left(v_{3}, v_{1}, v_{2}\right)$ and $\left(v_{2}, v_{3}, v_{1}\right)$ are the only other rotations determining this same cube and belonging to this component. Therefore the space of circumscribing cubes is homeomorphic to the orbit space of a cyclic group of order three operating on $S^{1}$. This group is generated by a rotation through $2 \pi / 3$. The orbit space is again $S^{1}$. The situation in higher dimensions is not known.

\section{BIBLIOGRAPHY}

1. S. S. Cairns, Circumscribing cubes in Euclidean n-space, Bull. Amer. Math. Soc. 65 (1959), 327-328.

2. - Umschriebende Wiirfel von konvexen Körpern in euklidischen Räumen, J. Reine Angew. Math. 208 (1961), 91-101.

3. - Differentiable approximations and the Schoenfies theorem for polyhedra, Mimeographed Report, University of Illinois, Urbana, 1963.

4. H. G. Eggleston, Convexity, Cambridge Tracts in Mathematics and Mathematical Physics, No. 47, Cambridge Univ. Press, New York, 1958.

5. A. Heller, On equivariant maps of spaces with operators, Ann. of Math. 55 (1952), 223-231.

6. S. Kakutani, $A$ proof that there exists a circumscribing cube around any bounded closed set in $R^{n}$, Ann. of Math. 43 (1942) 739-741.

7. A. N. Milgram, A solution of the frame problem for the three sphere, Abstract 213, Bull. Amer. Math. Soc. 56 (1950), 185.

8. R. S. Palais, Lectures on Morse theory, Mimeographed Notes, Harvard University, Cambridge, Mass., 1963.

9. N. Steenrod, The topology of fibre bundles, Princeton Univ. Press, Princeton, N. J., 1951.

10. H. Yamabe and $Z$. Yujobo, On the continuous function defined on a sphere, Osaka Math. J. 2 (1950), 19-22.

LEHIGH UNIVERSITY 